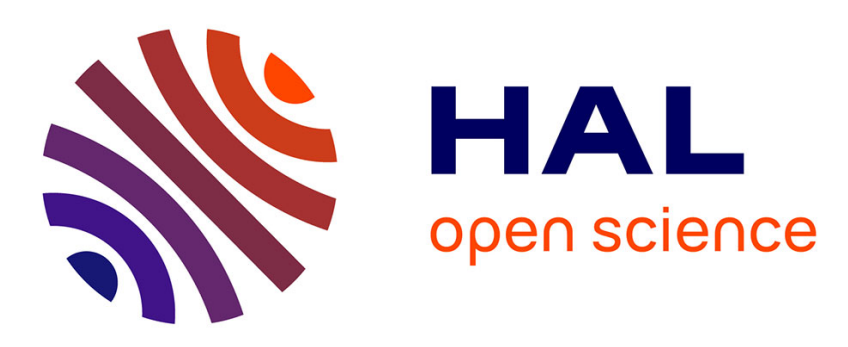

\title{
An Energy-Saving Speed Profile Algorithm for Cybernetic Transport Systems
}

Carlos Flores, Vicente Milanés, Joshué Pérez, Fawzi Nashashibi

\section{To cite this version:}

Carlos Flores, Vicente Milanés, Joshué Pérez, Fawzi Nashashibi. An Energy-Saving Speed Profile Algorithm for Cybernetic Transport Systems. ICVES 2015 - IEEE international conference on vehicular electronics and safety, Nov 2015, Yokohama, Japan. hal-01243298

\section{HAL Id: hal-01243298 \\ https://hal.inria.fr/hal-01243298}

Submitted on 14 Dec 2015

HAL is a multi-disciplinary open access archive for the deposit and dissemination of scientific research documents, whether they are published or not. The documents may come from teaching and research institutions in France or abroad, or from public or private research centers.
L'archive ouverte pluridisciplinaire HAL, est destinée au dépôt et à la diffusion de documents scientifiques de niveau recherche, publiés ou non, émanant des établissements d'enseignement et de recherche français ou étrangers, des laboratoires publics ou privés. 


\title{
An Energy-Saving Speed Profile Algorithm for Cybernetic Transport Systems
}

\author{
Carlos Flores, Vicente Milanés, Joshué Pérez, Fawzi Nashashibi
}

\begin{abstract}
Cybernetic Transport Systems (CTS) are now available on some cities worldwide covering the so-called lastmile for people transportation. These electric vehicles are expecting to operate during several hours without interruptions. It implies that energy consumption plays a key role when it comes to extend its autonomy. This paper presents a new algorithm for reference speed generation of CTS with energy consumption considerations. It consists on a two layer controller: the first one is in charge of generating the speed reference based on an energetic model, and the second one is a high-level fuzzy system to improve some low-level undesirable behaviors. The designed system was compared with three other algorithms in a real platform, showing promising results in terms of energy saving.
\end{abstract}

\section{INTRODUCTION}

The development of new automotive systems aid drivers not only on the safety-related driving aspects but also on improving users' quality of life. Efforts on this direction are mainly focused on the development of zero-emission vehicles. Autonomy is one of the key aspects to increase the market penetration of electric vehicles compared with gas-propelled ones, leading the change toward eco-friendly cities.

Among the different environments where vehicles drive, urban areas represent the most complex ones. This is because the constant interaction with other vehicles either at intersections, roundabouts or merging zones. One of the most promising urban areas for introducing fully automated vehicles is the so-called last-mile transportation sector [1]. It refers to the vehicles in charge of moving people from a transport hub-i.e. bus or train station-to their final destination. Cybernetic Transportation Systems (CTS) [2] have rose as the adequate solution to satisfy the last-mile sector requirements. CTSs are urban mobility platforms formed by electric-powered car-like robots. They are equipped with a set of sensors providing the ability of autonomously drive in urban areas [3].

CTSs have been actively investigated on recent years. There are currently some different solutions that have been developed to improve transportation network. Some of them are already working on public areas. An example of such systems is implemented over Heathrow London Airport, which consists on several driverless vehicles called Heathrow pods, which cover specified routes over one of the airport

Authors are with the Robotics and Intelligent Transportation Systems (RITS) Team, Inria Paris-Rocquencourt, Domaine de Voluceau, 78153 Le Chesnay, France \{carlos-eduardo.flores-pino, vicente.milanes, joshue.perez_rastelli,

fawzi.nashashibi\} dinria.fr terminal depending on the passenger desired destination. Additionally, there are several European projects dealing with the development of these transportation systems as Cybercars I and II, CitynetMobil or CityMobil I and II, among others. These projects carried out multiple demonstrations showing the CTS ability for moving people in urban areas.

CityMobil-2 project have demonstrated a fleet of CTS vehicles for covering last-mile routes in some European cities. Some of these demonstrations have been successfully carried out at Oristano (Italy) and at Leon (Spain). During these demonstrations, a shuttle service with several stops was installed in the cities, integrating new technologies to improve urban mobility and to determinate how people accepts these transportation systems.

The main objective of this solution is to provide a transportation service for people, being able to cover a pre-defined route constantly. Among the advantages of CTSs, they are able to travel over any unstructured environment without requiring a special infrastructure modification. However, one of the current limitations of CTS is coming from the fact of being electric cars, limiting the autonomy. Even though remarkable demonstrations have been carried out-i.e. vehicle fleet that covered urban routes as buses would, one of its main limitations is coming from the battery life. It is required that the service works with an uninterrupted schedule such as an urban bus does. The route used has been previously pre-defined, being always the same. It allows to optimize and reduce the vehicle energy consumption for each route.

This article aims to find a solution to the CTS vehicles autonomy problem by using an energy optimization algorithm. Considering a pre-defined route, the vehicle identifies the different stretches to be covered, modifying accordingly its speed with energy-saving purposes. One of the key factors is that the vehicle has to cover these routes in a fixed time that cannot be modified. This is used as input parameter to cover the pre-defined route, but with a monitoring on the energy consumption.

\section{UNDERSTANDING CYBERCARS}

CTSs or Cybercars are a new concept of fully automated electric vehicles with the purpose of creating an efficient transportation service on urban environments. They are composed of two main intelligence blocks: one that carries out the environment understanding and another for planning, decision and control. These blocks follow predefined trajectories without any driver intervention.

Cybercars have been designed aiming to create a lowcost transportation service for last-mile sector requirements, 


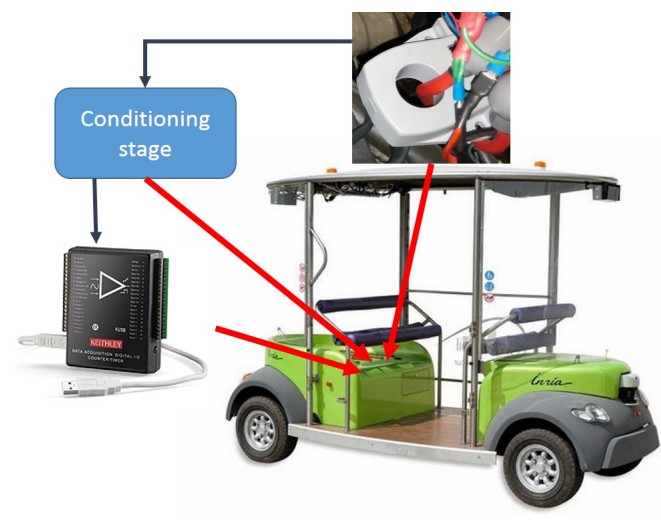

Fig. 1. Hall effect sensor, acquisition stage and the Analog Input to USB card required; and their place over the Cybus

which means that Cybercars are also low-cost platforms. Specifically, this work has used a Cybus (see Fig. 1) as experimental platform. It is a four-wheeled CTS prototype. It is equipped with two DC motor. One coupled to the wheel axis that is in charge of producing the torque required; and another coupled to the direction bar. A localization system -i.e. Simultaneous Localization and Mapping (SLAM)and multiple sensors-Inertial Measurement Unit (IMU), Encoders and Lasers-are used to provide the vehicle state.

\section{A. CTS Constraints}

As a consequence of being low-cost vehicles, they present some hardware constraints that can significantly affect their performance in comparison with regular vehicles. Below, the different limitations associated to the prototype cybercar used in this work-a Cybus-are detailed:

1) It is not equipped with a regenerative braking system. This causes that the embedded motor driver is not able to generate energy when driving downgrade.

2) The vehicle is propelled by a DC motor directly coupled to the wheel axis. It means that even in downgrades, the cybercar can consume energy.

3) When the vehicle brakes, a reverse torque is demanded to reduce the speed which is also causing an energy consumption.

4) On-board sensors-i.e. embedded encoders-are also low-cost devices with low resolution $(0.5 \mathrm{~m} / \mathrm{s})$. This fact affects both the optimal speed profile algorithm and the low-level control of the Cybus. Figure 2 shows the reference (red line) and measured speed (blue line), and the instant power consumed (green line), illustrating this problem. It shows how the reference speed is not being correctly followed by the lowlevel controller. This behavior is directly translated on an undesired energy consumption because of the compensation-that is, torque applied backwards to slow down the vehicle-carried out by the controller to follow the reference.

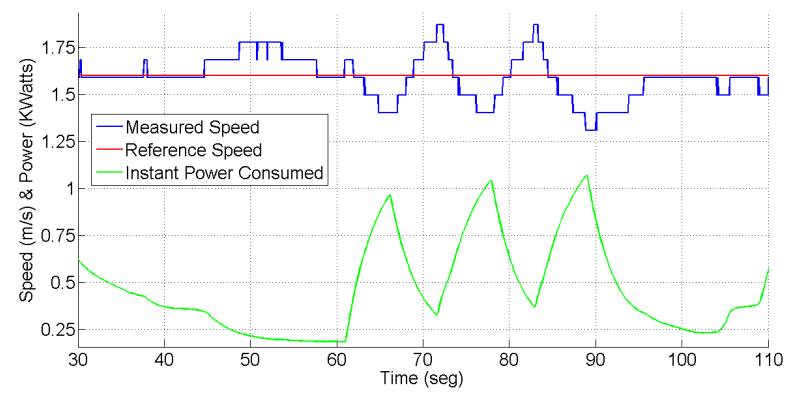

Fig. 2. Example of speed low level control problems.

\section{B. Proposed Algorithm}

This paper considers the scenario of a Cybercar that works as a last-mile transportation service, covering a specific route between two points all day long. This work aims to solve the autonomy problem and extend the battery life when the vehicle is driving, maintaining the pre-defined route and taking the same time to cover it.

The proposed solution is an improved algorithm based on a previous RITS work [4]. It depends on both the route and vehicle characteristics. It is also proposed a highlevel fuzzy system to deal with the constraints previously described; mainly, the low-level control that causes undesired power peaks which are translated on energy losses, reducing vehicle's autonomy.

\section{SPEED PROFILE GENERATION}

This section describes the optimal speed profile algorithm to increase vehicle's autonomy. Its goal is to send the optimal reference speed to the low-level control in function of the road slope, the vehicle properties and the fixed time to cover the route.

Figure 3 illustrates how the optimal speed profile algorithm is coupled to the control architecture, including the two main blocks: Partial and Fully Profile Generation. The former is in charge of splitting the whole route into segments according to their slope in order to estimate the energy that the vehicle will expend while traveling along the route. This calculation is done before the vehicle departure. Since the required instant power is calculated for each segment, its optimal speed can be obtained through the energetic model.

Once the cybercar starts its route, the algorithm constantly requires the current position in order to set the appropriate reference speed according to the road layout (speed profile obtained in the Partial Profile Generation). This block is also in charge of executing the speed transitions from one segment to the next one through the generation of speed ramps. Finally, the reference speed is sent to the low-level controller to move the motor driver according to the reference.

\section{A. High-level fuzzy system}

This high-level controller is implemented to modify the output of the Partial Profile Generation output in order to achieve a more efficient driving. The motivation of selecting 


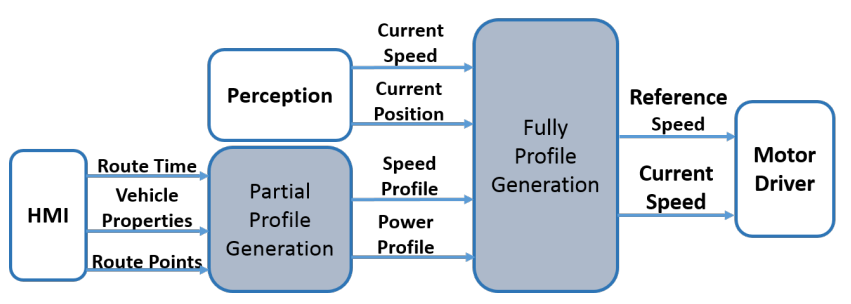

Fig. 3. Algorithm block diagram

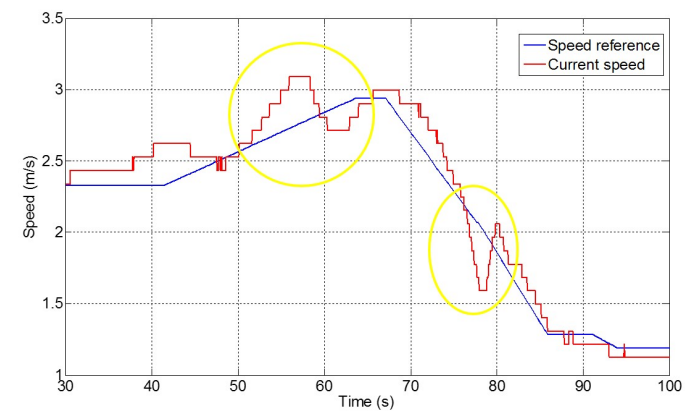

Fig. 4. Low-level unappropriated behavior

a fuzzy controller for this work is mainly based on two reasons: 1) its ability of applying human reasoning to its tuning [5]; and 2) the fact that a fuzzy controller does not require a mathematical model, which is appropriate in this case due to the difficulty of modeling the power-speed behavior of the vehicle [6].

Figure 4 shows how the low-level speed controller behaves. The failures on its response are highlighted on yellow circles. The real measured speed oscillates around a reference given, which is translated to more energy consumption when trying to correct the speed error. The fact that the low level speed control was factory made, brings as a consequence that the controller tuning values are unreachable and their modifications are out of the bounds of this work.

The reason for introducing a high-level fuzzy controller is for dealing with the unappropriated behavior of the lowlevel controller. The proposed fuzzy controller takes into consideration two inputs: Speed error (in meters per second), defined as measured speed minus reference one, and Power error (in KWatts) defined as measured instant power minus current optimal power. The former avoids the excessive braking maneuver when the error between the actual and desired speed is low. The latter is in charge of trying to follow the maximum optimal energy consumption.

RITS team at INRIA has developed a fuzzy software called FEMOT (Fuzzy Embedded Motor) which is an inference motor with a natural language-based input language [5]. Specifically for this application, FEMOT operates using Mamdani's inference method, with singleton-type membership functions to codify the output variables, taking control actions with high accuracy and very short delays which are key factors for real-time control systems.

The Fuzzy logic controller is defined through the rule base presented on table I. A heuristic method was followed
TABLE I

CONTROLler RULE BASE

\begin{tabular}{|c|c|c|c|}
\hline \multirow{2}{*}{ PowerError } & \multicolumn{3}{|c|}{ SpeedError } \\
\cline { 2 - 4 } & Positive & Null & Negative \\
\hline Positive & -0.2 & 0 & 0.2 \\
\hline Null & -0.1 & 0 & 0.1 \\
\hline Negative & 0.7 & 0 & -0.6 \\
\hline
\end{tabular}

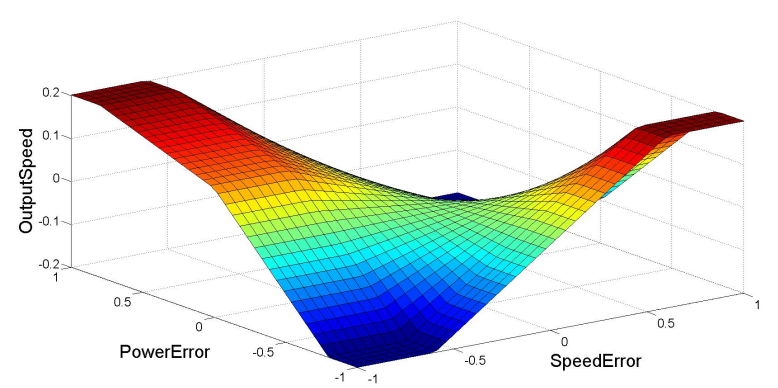

Fig. 5. Fuzzy control surface

to define the controller according to human reasoning and driver's needs, taking advantage of fuzzy logic control. Several tests showed that the situations in which the power error is negative (downgrade segments) are the ones that cause the low-level control failures. For this reason, the highlevel controller has been designed to modify the reference speed, following the measured one. This action avoids that the low level could appreciate a great speed error and, as a consequence, the execution of a correction procedure as well. On the other hand, the positive speed error case requires a common controller behavior, such as increasing the reference speed when the error is getting negative as example.

Finally, the high level fuzzy controller describes a control surface as shown on figure 5. It shows the smoothness through the whole surface, validating the suitability of the fuzzy rules selected.

Figure 6 shows how the high-level fuzzy controller is coupled to the control architecture. The reference speed is generated by adding the algorithm and the controller outputs. In this way, the low level is able to receive the control commands from the high-level, acting in a more efficient way.

\section{EXPERIMENTAL RESULTS}

The proposed algorithm was validated using an experimental platform at Inria's facilities. The selected scenario includes upgrade, flat and downgrade segments to evaluate the performance of the system in different layouts. The upper part of Figure 7 shows an aerial view with the selected route remarked on yellow whereas the bottom part depicts the elevation profile of the entire course. The reference speed, the real speed and the instant power consumed was measured in real time on each travel.

\section{A. Comparative analysis}

Using the trajectory showed on Fig. 7, four driving behavior were defined to compare the performance of the 


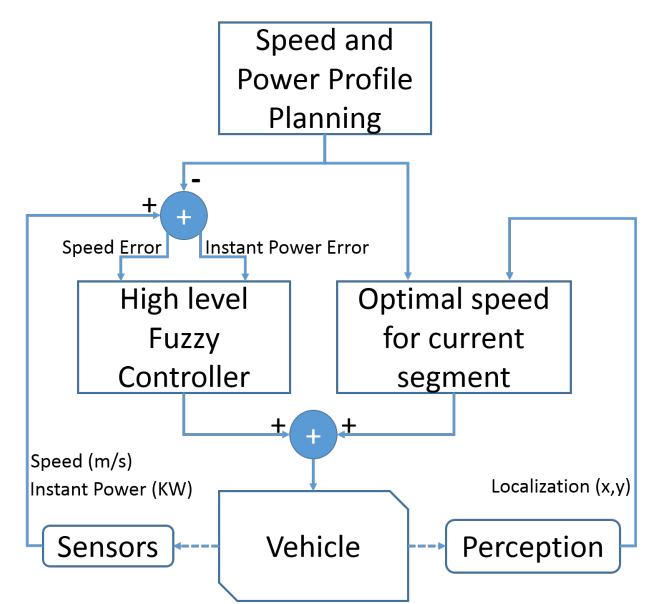

Fig. 6. Fully Speed Generation Architecture

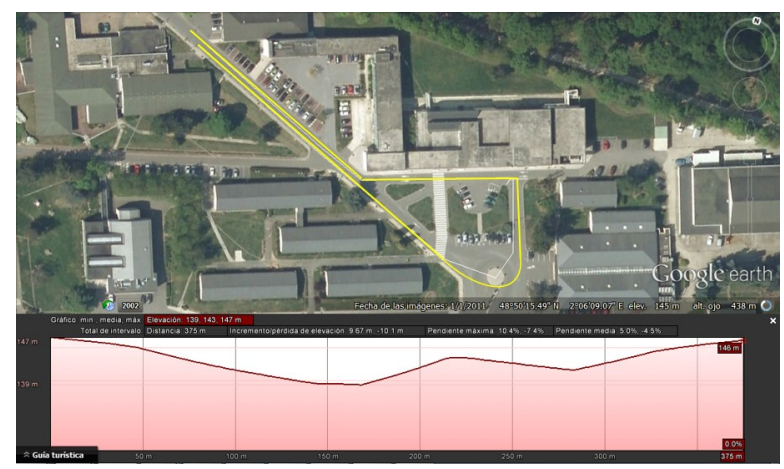

Fig. 7. Test route at Inria's facilities

developed system with current speed control algorithms. The first test is the one explained on section II-B, where a commuter drives manually a vehicle trying to do it efficiently from a starting point to a destination. The second test shows the performance of a vehicle equipped with a cruise control system. The third case is when the intelligent speed profile algorithm is applied over the longitudinal control, to check the energy consumption. Finally, the fourth scenario applies the same algorithm, but adding the high-level fuzzy controller to improve the reference speed generation. It is important to remark that the conditions for every scenario are the same, either the trajectory or the time established to cover the route. On each of these results, the reference speed (red line), the measured speed (blue line) and the instant power consumed (green line) are plotted.

1) Manual Driving: Figure 8 illustrates the reference speed generated by the driver to the low level control, the measured speed and the instant power consumed by the vehicle. The power consumed presents multiple peaks, increasing the energy consumption. This is because the continuous action over the acceleration/braking interfaces of the vehicle when trying to follow the reference speed.

2) Cruise Control: As second case, a cruise control system was tested. It maintains the reference speed constant during the whole route. Figure 9 shows the result of this test. As it can be appreciated, the power consumed turns to be
TABLE II

ENERGY CONSUMPTION PER ALGORITHM

\begin{tabular}{|c|c|c|}
\hline $\begin{array}{c}\text { Evaluation } \\
\text { Scenario }\end{array}$ & $\begin{array}{c}\text { Consumed Energy } \\
\text { (KJoules) }\end{array}$ & $\begin{array}{c}\text { Savings } \\
\text { Percentage (\%) }\end{array}$ \\
\hline Manual Driving & 243.23 & - \\
\hline Cruise Control & 213.74 & 12.12 \\
\hline ISP Algorithm & 201.68 & 17.08 \\
\hline ISPA + HL Fuzzy Controller & 188.07 & 22.68 \\
\hline
\end{tabular}

more stable although there are still undesirable power peaks and some stretches where the energy consumption is higher than it could be.

3) Speed Profile Algorithm: The route described on figure 7 was divided in different segments in order to apply the Intelligent Speed Profile Algorithm (ISPA). It permits to determine the optimal speed for each of the different stretches that the vehicle is covering through the route. Figure 10 shows how the reference speed changes along the trajectory, trying to pursuit the best energy consumption over every road layout. There can also be seen that the power peaks caused by the low level control have been significantly eliminated but there are still some of them present on the downgrade stretch.

4) Speed Profile Algorithm + High Level Fuzzy Controller: Figure 11 plots the result of adding the high level fuzzy controller to the ISPA. One can see how the new reference speed-formed by adding the algorithm output plus the fuzzy controller output-is modified according to the segment slope, fluctuating around the reference generated by the algorithm for avoiding peaks in downgrades. This modification causes by the fuzzy controller avoids the correction executed by the low-level, leading to a great energy saving.

\section{B. Results Analysis}

Table II shows a comparison between the energy saving of each of the proposed algorithm. It is interesting to notice that Cruise Control systems significantly improved energy consumption with respect to a human driver. However, no specific behavior for energy saving is carried out since the reference is set to the same value either during upgrades or downgrades. The proposed ISPA system brings this consideration into the equation, generating a specific reference speed for each of the stretch in the route. It causes an improvement in the energy consumption on the part of the Cybus. Finally, the high-level controller specifically designed to deal with the low-level problems improves all the previous algorithms.

The power peaks that were appreciated on the first, second and third case were totally removed by adding the high level fuzzy controller, which acts on downgrades reducing the speed error, to prevent a braking action from the low level to the motor. Otherwise, when the vehicle is going through an upgrade segment, the fuzzy controller corrects normally the error.

The comparison from Table II shows a saving percentage of $22.68 \%$ comparing the manually driven tests with the proposed algorithm. The amount of energy consumed was determined by integrating the instant power consumed while 


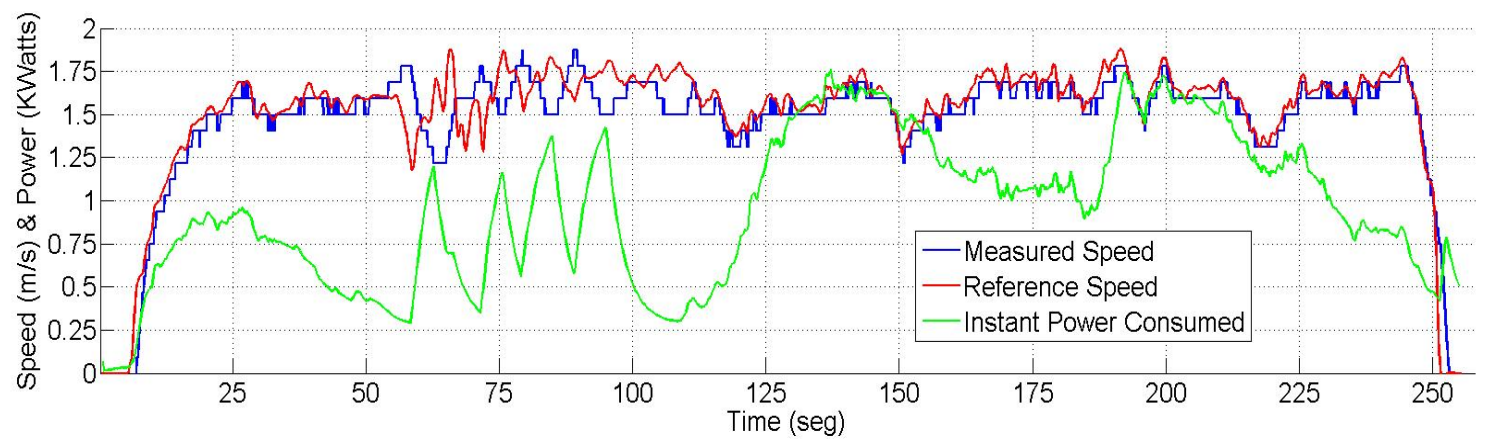

Fig. 8. Manual driver test

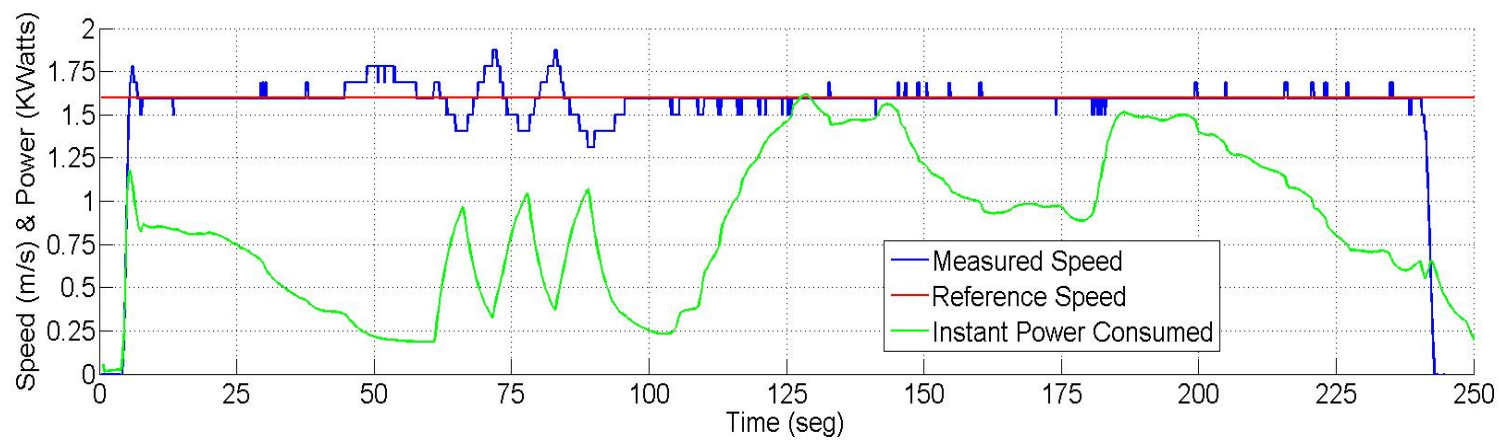

Fig. 9. Cruise Control test

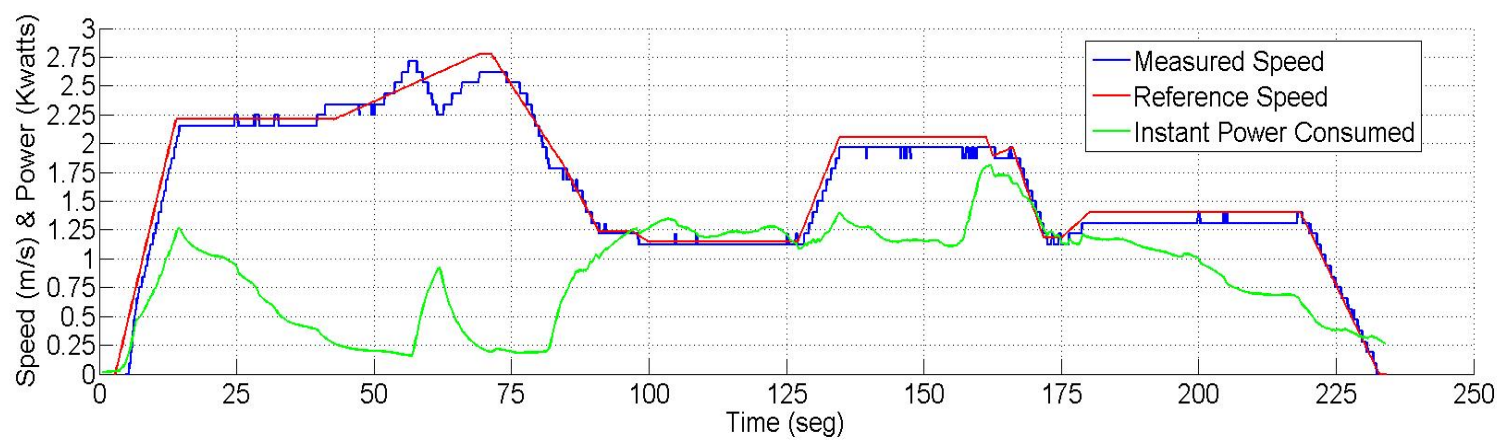

Fig. 10. ISPA test

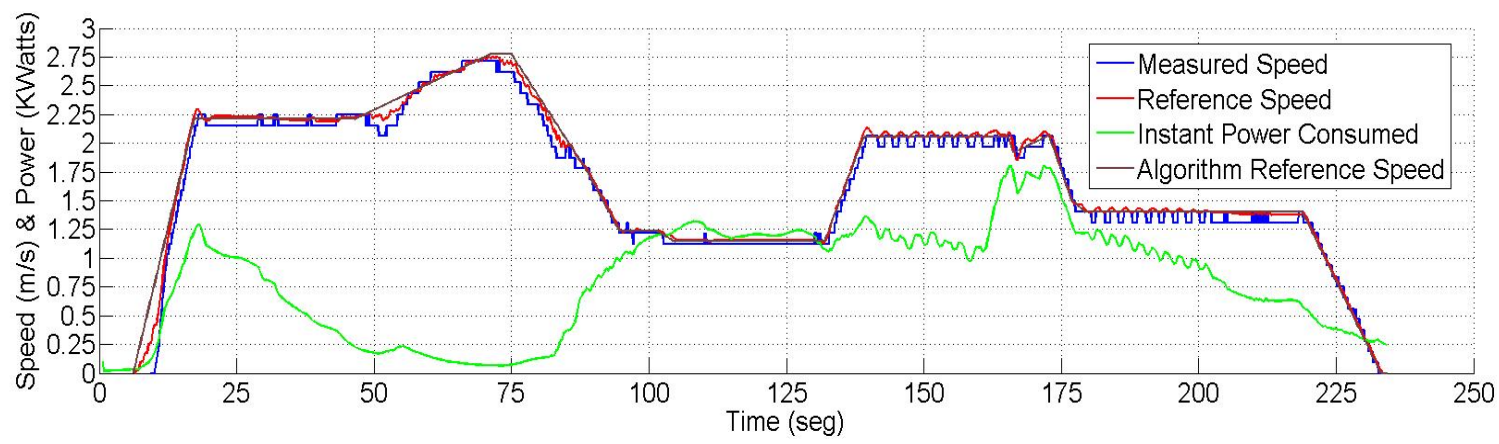

Fig. 11. ISPA + fuzzy algorithm test 


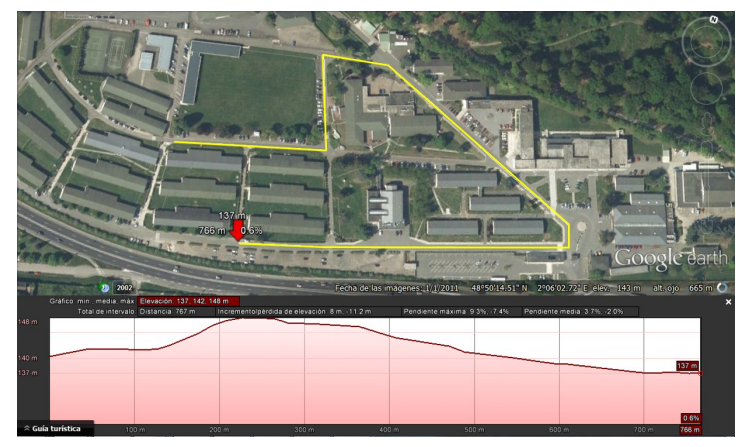

Fig. 12. Validation route

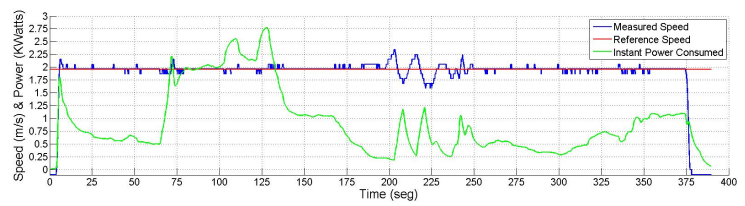

Fig. 13. $\mathrm{CC}$ behavior on the validation route

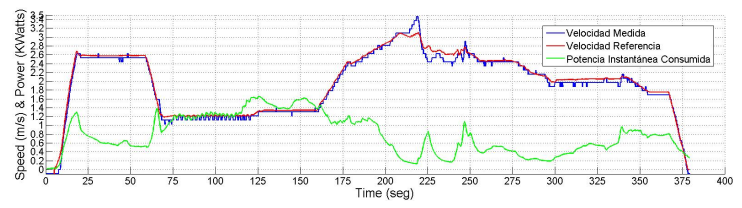

Fig. 14. ISPA+fuzzy behavior on the validation route

the vehicle was traveling along the route on each of the scenarios.

\section{Validation}

Another route was selected in order to test the performance of the module developed using a different layout that the one used for adjusting the fuzzy controller. The new trajectorysee Figure 12-is formed by several upgrade, flat and downgrade segments. It has a total length of 766 meters, a top slope of $9.3 \%$ and a minimum slope of $-7.4 \%$, being different enough from the first scenario.

1) Result Analysis: Using the route presented on Fig. 12, two algorithms were set to compare the performance of the developed work. The first shows a car equipped with a cruise control system would cover the route (see Fig. 13 ), keeping the speed constant. The second case is where the proposed architecture is applied: the intelligent speed profile algorithm with the high level fuzzy controller generating the reference speed given to the low level (see Fig. 14).

Figure 14 exhibits some power peaks appear when the Cybus goes downgrade. This is because the length of the segment. The vehicle reaches the maximum allowed speed, causing a braking action from the low-level controller. Table III presents the energy consumption on each case, showing the big saving using the proposed algorithm compared with the classical cruise control systems.
TABLE III

ENERGY CONSUMPTION ON THE VALIDATION ROUTE

\begin{tabular}{|c|c|c|}
\hline $\begin{array}{c}\text { Evaluation } \\
\text { Scenario }\end{array}$ & $\begin{array}{c}\text { Consumed Energy } \\
\text { (KJoules) }\end{array}$ & $\begin{array}{c}\text { Savings } \\
\text { Percentage (\%) }\end{array}$ \\
\hline Cruise Control & 355.03 & - \\
\hline ISPA + HL Fuzzy Controller & 297.12 & 16.31 \\
\hline
\end{tabular}

\section{CONCLUSions}

This paper presents an intelligent control algorithm for reference speed generation, considering energy consumption. An energetic model able to generate the proper speed for each segment on the route was described. Additionally, a fuzzy controller is proposed to improve low-level limitations on the experimental platform.

The proposed method was compared with three other algorithms, showing its benefits in terms of energy savings. All algorithms was compared using the same road layout and the same time to cover the route. Results demonstrated that the proposed system significantly extends the battery life of CTS, showing promising results for future implementations on fleets.

As future works, real time traffic information will be considered in order to recalculate the reference speed on each segment on real time.

\section{ACKNOWLEDGMENTS}

Authors express their gratitude to the CoCoVeA (Coopération Conducteur-Vehicule Automatisé) ANR project for its support in the development of this work.

\section{REFERENCES}

[1] Z. Chong, B. Qin, T. Bandyopadhyay, T. Wongpiromsarn, E. Rankin, M. Ang, E. Frazzoli, D. Rus, D. Hsu, and K. Low, "Autonomous personal vehicle for the first- and last-mile transportation services," in Cybernetics and Intelligent Systems (CIS), 2011 IEEE 5th International Conference on, 2011, pp. 253-260.

[2] M. Parent, G. Gallis, A. Alessandrini, and T. Chanard, "Cybercars: Review of first projects," Inria,University of Rome and GEA- Lausanne, Tech. Rep., 2003.

[3] S. Boisse, R. Benenson, L. Bouraoui, M. Parent, and L. Vlacic, "Cybernetic transportation systems design and development: Simulation software," in ICRA Workshop: Planning, Perception and Navigation for Intelligent Vehicles, 2007.

[4] C. Flores, V. Milanés, J. Pérez, D. González, and F. Nashashibi, "Optimal energy consumption algorithm based on speed reference generation for urban electric vehicles," 2015 IEEE Intelligent Vehicles Symposium, 2015.

[5] M. O. Rojas, J. P. Rastelli, N. Fawzi et al., "Fuzzy logic techniques for cybercars: a control and decision approach." in 22èmes rencontres francophones sur la Logique Floue et ses Applications (LFA 2013), 2013.

[6] J. Perez, V. Milanés, and E. Onieva, "Cascade architecture for lateral control in autonomous vehicles," IEEE Transactions on Intelligent Transportation Systems, vol. 12, no. 1, pp. 73-82, 2011. 